\title{
The Effects of Repetitive Transcranial Magnetic Stimulation on the Gait of Acute Stroke Patients
}

\author{
Sang-Goo $\mathrm{Ji}^{1}$ and Myoung-Kwon Kim ${ }^{2 *}$ \\ ${ }^{1}$ Department of Physical Therapy, Eulji University Hospital, Dunsan-dong, Seo-gu, Daejeon 302-799, Korea \\ ${ }^{2}$ Department of Physical Therapy, College of Rehabilitation Sciences, Daegu University, Gyeongsan-si 712-714, Korea
}

(Received 2 April 2015, Received in final form 13 May 2015, Accepted 14 May 2015)

\begin{abstract}
The aim of the present study was to examine whether repetitive transcranial magnetic stimulation (rTMS) can improve gait ability of acute stage stroke patients. This study was conducted with 39 subjects who were diagnosed as having a hemiparesis due to stroke. The experimental group included 20 subjects who underwent repetitive transcranial magnetic stimulation and the control group included 19 subjects who underwent sham therapy. The stroke patients in the experimental group underwent conventional rehabilitation therapy and rTMS was applied daily to the hotspot of the lesional hemisphere. The stroke patients in the control group underwent sham rTMS and conventional rehabilitation therapy. Participants in both groups received therapy five days per week for four weeks. Temporospatial gait characteristics, such as stance phase, swing phase, step length in affected side, velocity, and cadence, were assessed before and after the four week therapy period. A significant difference was observed in post-treatment gains for the step length in the affected side, velocity, and cadence between the experimental group and control group $(p<0.05)$. However, no significant differences were observed between the two groups on stance phase and swing phase $(p>0.05)$. We conclude that rTMS may be beneficial in improving the effects of acute stage stroke on gait ability.
\end{abstract}

Keywords : acute stroke, gait, repetitive transcranial magnetic stimulation

\section{Introduction}

Stroke is one of the principal causes of morbidity and mortality in adults in the developed world and the leading cause of disability in all industrialized countries. Impairment of gait ability is one of the major devastating outcomes of post-stroke sequel. After a stroke, gait speed, cadence, and swing phase decrease, whereas gait cycle duration and stance phase increase [1]. Energy expenditure during walking is higher in patients with hemiparesis than in people who are healthy, and variability in oxygen consumption after a stroke reflects gait deviations [2]. Therefore, gait restoration has been recognized as a primary goal in stroke rehabilitation.

Transcranial magnetic stimulation (TMS) of the human motor cortex represents a non-invasive method for evaluating corticospinal influence during natural movements. TMS excites the corticospinal system, producing a

CThe Korean Magnetics Society. All rights reserved.

*Corresponding author: Tel: +82-53-850-4352

Fax:+82-53-850-4359, e-mail: kimmk@daegu.ac.kr short-latency excitation of spinal motoneurons [3]. Repetitive TMS (rTMS) is a series of magnetic pulses that temporarily summate and change neural activities to a greater degree than traditional single-pulse TMS. According to recent rTMS research, the primary motor cortex can reorganize and modulate the interactions between the ipsilesional and contralesional motor cortex following a stroke [4]. According to findings from recent studies on the safety of high-frequency rTMS, a rTMS at a subthreshold intensity of $20 \mathrm{~Hz}$ was proposed as safe and beneficial to motor function. In a previous study, we found that a single session of $10 \mathrm{~Hz}$ rTMS at subthreshold intensity facilitated practice-dependent plasticity and improved motor learning in patients with chronic stroke [5]. rTMS in early rehabilitation can produce a more powerful effect on neural plasticity, and subsequent behavioral changes last longer.

However, studies of rTMS have mainly addressed subacute or chronic stage stroke patients. Therefore, this study was conducted to administer rTMS to acute stage patients within 3 months after a stroke when neurological recovery occurs and to examine the effects of rTMS on 
the patients' gait ability.

\section{Materials and Methods}

This study was conducted with 39 subjects who were diagnosed as having a hemiparesis due to stroke. Sufficient explanation of this study's intention and the overall purpose is given, and voluntary consent to participate in this study was obtained from all subjects. All procedures were reviewed and approved by the Institutional Ethics Committee in the Eulji University Hospital. They were randomly assigned to either the experimental group of 20 subjects or the control group of 19 subjects. Inclusion criteria for the participation of this study were as follows: (1) stroke onset duration of $<3$ months, (2) no neurological deficits in cerebellum or the brainstem, (3) the ability to walk at least $10 \mathrm{~m}$ without assistance, (4) no unilateral neglect, hemianopsia, or apraxia, and (5) no psychological or emotional problems.

Exclusion criteria were as follows: (1) patients with metal within the brain, (2) patients with a cardiac pacemaker, (3) pregnant women, and (4) a history of seizure. The demographic information of the subjects is summarized in Table 1. The subjects in the experimental group received rTMS and conventional rehabilitation therapy for a total of 50 minutes (rTMS: 20 minutes; conventional rehabilitation therapy: 30 minutes) per day, with a 10 minute rest period halfway through the session. The subjects in the experimental group received training five days per week for four weeks. Conventional rehabilitation therapy consists of neural development treatment. The subjects in the control group received sham rTMS and conventional rehabilitation therapy for a total of 50 minutes (sham rTMS: 20 minutes; conventional rehabilitation therapy: 30 minutes) per day on the same days.

A 70-mm figure 8 coil and a Magstim Rapid stimulator (Magstim Company, Dyfed, UK) were used for both groups. For patients in the experimental group, $10 \mathrm{~Hz}$ rTMS was applied daily to the hotspot of the lesional hemisphere in 10 -second trains, with 50 -second intervals between trains, for 20 minutes (total 2,000 pulses). The control group performed sham rTMS for the same duration as the experimental group.

Temporospatial gait data were obtained using a sixcamera motion analysis system (Eagle system, Motion Analysis, Santa Rosa, CA, USA), sampling at $120 \mathrm{~Hz}$. Hemispherical or flat reflective markers $(25 \mathrm{~mm}$ diameter) were applied at the following sites on both sides of the body: anterior superior iliac spine, superior aspect of the L5-sacral interface, thigh (lower thigh below the midpoint), medial femoral condyle, lateral femoral condyle,
Table 1. General and Medical Characteristics of Subjects $(\mathrm{N}=39)$

\begin{tabular}{lcc}
\hline \hline & EG $(\mathrm{n}=20)$ & CG $(\mathrm{n}=19)$ \\
\hline Age (year) & $55.65(8.95)^{\mathrm{a}}$ & $56.36(10.44)$ \\
Height $(\mathrm{cm})$ & $165.25(6.02)$ & $164.47(7.00)$ \\
Weight $(\mathrm{kg})$ & $66.45(8.53)$ & $68.42(6.15)$ \\
Since onset (Month) & $1.90(0.72)$ & $1.68(0.58)$ \\
Gender (Male/Female) & $11 / 9$ & $12 / 7$ \\
Affected side (Left/Right) & $9 / 11$ & $10 / 9$ \\
Type of stroke & $13 / 7$ & $14 / 5$ \\
(Ischemia/Hemorrhage) & & $27.05(1.96)$ \\
MMSE-K (Score) & $26.85(2.08)$ & \\
\hline
\end{tabular}

${ }^{\mathrm{a}}$ mean (SD)

EG: rTMS and conventional rehabilitation therapy

CG: Sham rTMS and conventional rehabilitation therapy

shank (lower shank below the mid-point), medial malleolus, lateral malleolus, posterior calcaneus, and center of the foot between the $2^{\text {nd }}$ and $3^{\text {rd }}$ metatarsal. To facilitate application and visualization of the markers, the subjects wore a pair of skin-tight cycling shorts and a sleeveless top.

EvaRt and Orthotrak software (Motion Analysis, Santa Rosa, CA, USA) were used for processing the data. After a test walk to become accustomed to the experimental procedure, the subjects were asked to walk along a $7 \mathrm{~m}$ walkway for three sessions, and the average values of the data were used. We evaluated the temporospatial gait characteristics, such as the stance phase, swing phase, step length, velocity, and cadence of the experimental group and the control group using a motion analysis device [6].

For this study, the subjects were instructed to stand in front of the gait board, and then walk it in a self-selected comfortable speed until they reached the end of the board. Differences in the general characteristics between the experimental group and the control group before the intervention were compared using independent t-tests and chi-square tests. Comparisons of gait before and after the intervention within each group were made using a paired samples t-test. Comparisons of pre- and post-test differences in gait between the experimental group and the control group were made using the independent t-test. The statistical software SPSS 18.0 (SPSS, Chicago, IL, USA) was used for statistical analysis. The level of significance was chosen as 0.05 .

\section{Results}

The values of the gait ability of both groups are summarized in Table 2. Significant differences were observed 
Table 2. Comparison of change in temporospatial gait characteristics between each group ( $N=39)$

\begin{tabular}{|c|c|c|c|c|c|c|}
\hline & \multicolumn{3}{|c|}{$\mathrm{EG}(\mathrm{n}=20)$} & \multicolumn{3}{|c|}{$\mathrm{CG}(\mathrm{n}=19)$} \\
\hline & Pre & Post & CWG & Pre & Post & CWG \\
\hline Stance phase in both sides (\%) & $67.90(4.51)^{\mathrm{a}}$ & $64.60(4.41)^{*}$ & $0.96(1.29$ to 5.31$)$ & $67.74(3.72)$ & $66.47(3.95)$ & $0.77(-0.36$ to 2.88$)$ \\
\hline Swing phase in both sides (\%) & $32.10(4.51)$ & $35.40(4.41)^{*}$ & $0.96(-5.31$ to -1.29$)$ & $32.26(3.72)$ & $33.52(3.95)$ & $0.77(-2.88$ to 0.36$)$ \\
\hline Step length in affected side $(\mathrm{cm})^{\dagger}$ & $30.55(4.72)$ & $38.75(8.66)^{* *}$ & $1.46(-11.27$ to -5.13$)$ & $31.21(4.84)$ & $33.42(4.56)^{* *}$ & $0.56(-3.39$ to -1.03$)$ \\
\hline Velocity $(\mathrm{cm} / \mathrm{s})^{\dagger}$ & $47.15(13.67)$ & $60.85(11.52)^{* *}$ & $2.83(-19.62$ to -7.78$)$ & $48.53(13.68)$ & $51.26(13.90)^{*}$ & $1.03(-4.91$ to -0.56$)$ \\
\hline Cadence (step/min) ${ }^{\dagger}$ & $69.55(9.26)$ & $81.00(8.60)^{* *}$ & $1.81(-15.24$ to -7.66$)$ & $70.32(12.85)$ & $72.00(13.15)^{*}$ & $0.61(-2.97$ to -0.39$)$ \\
\hline
\end{tabular}

${ }^{a}$ mean (SD), within group: ${ }^{*} p<0.05$, between groups: ${ }^{\dagger} p<0.05$, CWG: changes within groups

EG: rTMS and conventional rehabilitation therapy

CG: Sham rTMS and conventional rehabilitation therapy

between the post-test results of step length in affected side, velocity, and cadence of the two groups $(p<0.05)$. In the experimental group, significant differences were found in the pre- and post-test results of all variables $(p<0.05)$, whereas in the control group, a significant difference was observed only between the pre- and posttest results of step length on the affected side, velocity, and cadence $(p<0.05)$.

\section{Discussion}

This study was conducted to investigate the effect of rTMS applied for the improvement of gait ability in patients with acute stage stroke. According to the results of this study, the step length, velocity, and cadence of the experimental group were more enhanced than those of the control group after the intervention. rTMS was shown to be more effective in improving the gait ability than sham rTMS.

The ratio of swing phases in the experiment increased more in the experimental group than in the control group. Thus, the stability of the lower extremities and the trunk increased during walking. Moreover, the step length in the affected side of the experimental group is thought to have increased more than the control group because the ability to control the lower extremities and the trunk was improved through rTMS.

TMS has enabled teams to study the changes in the excitability of the primary motor cortices of the injured and healthy hemispheres during the recovery phase as well as the modulation of interhemispheric inhibition at rest and when planning and executing a voluntary movement [7]. Lomarevet al. [8] demonstrated that highfrequency rTMS has been shown to have a benefit for improving gait speed in brain injury. Yang et al. [9] investigated the effect of $5 \mathrm{~Hz}$ rTMS followed by treadmill training on cortical excitability as measured through TMS in 20 patients with brain injury, and examined whether normalizing cortical excitability was accompanied by an improvement in gait performance. The results showed an enhanced effect of treadmill training on the modulation of corticomotor inhibition and the improvement of walking speed and dynamic balance. Khedr et al. [10] showed that $5 \mathrm{~Hz}$ rTMS, with 2000 pulses per day for 10 days improved the walking speed and self-assessment of patients with brain injury.

In clinical studies, patterns of post-stroke recovery reported in the rehabilitation literature support the model derived from animal-based trials. For most individuals receiving stroke rehabilitation, optimal recovery of motor function and activities of daily living (ADL) is achieved soon after stroke onset. Jorgensen et al. [11] reported that the best neurological recovery was achieved within 11 weeks for $95 \%$ of patients admitted to a large urban center. Recovery of ADL function tended to occur more slowly, but at 12.5 weeks post-stroke, optimal recovery was achieved by most patients. The time course of functional recovery in rehabilitation is strongly influenced by the severity of the stroke as well as the severity of initial functional impairment.

These results support the perceived benefit of rTMS to augment on gait ability of acute stage patients within the first 3 months of a stroke event. The present study has some limitations. First, the small sample size may have influenced certain variables and impacted the results. Therefore, these results cannot be generalized to all stroke patients. Second, the absence of follow-up after the end of the rTMS does not allow for determination of the durability of the effect of this intervention. Further studies, including a long-term follow-up assessment, are needed to evaluate the long-term benefits of rTMS.

\section{References}

[1] K. Hill, P. Ellis, J. Bernhardt, P. Maggs, and S. Hull, Aust. J. Physiother. 43, 173 (1997). 
[2] A. Danielsson, C. Willen, and K. S. Sunnerhagen, Arch. Phys. Med. Rehabil. 88, 1298 (2007).

[3] J. C. Rothwell, P. D. Thompson, B. L. Day, S. Boyd, and C. D. Marsden, Exp. Physiol. 76, 159 (1991).

[4] C. Calautti, M. Naccarato, P. S. Jones, N. Sharma, D. D. Day, A. T. Carpenter, E. T. Bullmore, E. A. Warburton, and J. C. Baron, Neuroimage. 34, 322 (2007).

[5] Y. H. Kim, S. H. You, M. H. Ko, and J. W. Park, Stroke. 37, 1471 (2006).

[6] Y. R. Yang, Y. C. Chen, and C. S. Lee, Gait Posture. 25, 185 (2007).

[7] P. Cicinelli, R. Traversa, and P. M. Rossini, Electroen- cephalogr Clin. Neurophysiol. 105, 438 (1997).

[8] M. P. Lomarev, S. Kanchana, W. Bara-Jimenez, M. Lyer, E. M. Wassermann, and M. Hallett, MovDisord. 21, 325 (2006).

[9] Y. R. Yang, C. Y. Tseng, S. Y. Chiou, K. K. Liao, S. J. Cheng, K. L. Lai, and R. Y. Wang, Neurorehabil. 27, 79 (2013).

[10] E. M. Khedr, H. M. Farweez, and H. Islam, Eur. J. 10, 567 (2003).

[11] H. S. Jorgensen, H. Nakayama, H. O. Raaschou, J. ViveLarsen, M. Stoier, and T. S. Olsen, Arch. Phys. Med. Rehabil. 76, 406 (1995). 